\title{
Improvement of gross theory of beta-decay for application to nuclear data
}

\author{
Hiroyuki Koura ${ }^{1, a}$, Tadashi Yoshida ${ }^{2}$, Takahiro Tachibana ${ }^{3}$, and Satoshi Chiba ${ }^{2}$ \\ 1 Advanced Science Research Center, Japan Atomic Energy Agency, Shirakata 2-4, Tokai, Naka-gun, Ibaraki 319-1195, Japan \\ 2 Laboratory for Advanced Nuclear Energy, Tokyo Institute of Technology, Ookayama 2-12-1, Meguro-ku, Tokyo 152-8550, Japan \\ 3 Senior High School of Waseda University, Kamishakujii 3-31-1, Nerima-ku, Tokyo 177-0044, Japan
}

\begin{abstract}
A theoretical study of $\beta$ decay and delayed neutron has been carried out with a global $\beta$-decay model, the gross theory. The gross theory is based on a consideration of the sum rule of the $\beta$-strength function, and gives reasonable results of $\beta$-decay rates and delayed neutron in the entire nuclear mass region. In a fissioning nucleus, neutrons are produced by $\beta$ decay of neutron-rich fission fragments from actinides known as delayed neutrons. The average number of delayed neutrons is estimated based on the sum of the $\beta$-delayed neutron-emission probabilities multiplied by the cumulative fission yield for each nucleus. Such a behavior is important to manipulate nuclear reactors, and when we adopt some new high-burn-up reactors, properties of minor actinides will play an important roll in the system, but these data have not been sufficient. We re-analyze and improve the gross theory. For example, we considered the parity of neutrons and protons at the Fermi surface, and treat a suppression for the allowed transitions in the framework of the gross theory. By using the improved gross theory, underestimated half-lives in the neutron-rich indium isotopes and neighboring region increase, and consequently follow experimental trend. The ability of reproduction (and also prediction) of the $\beta$-decay rates, delayed-neutron emission probabilities is discussed. With this work, we have described the development of a programming code of the gross theory of $\beta$-decay including the improved parts. After preparation finished, this code can be released for the nuclear data community.
\end{abstract}

\section{Introduction}

The $\beta^{-}$decay occurs with a weak interaction in the neutron-rich nuclear mass region and nucleus releases electron, gamma-ray and anti-neutrino. Many neutronrich nuclei can emit neutrons through $\beta$ decay, called delayed neutrons. Delayed neutrons play an important role in allowing nuclear reactors to be safely controlled, due to the delay that occurs between neutron-induced fission events and their eventual emission.

In order to estimate $\beta$-decay rate and delayed neutron probability theoretically, calculation of the nuclear matrix elements of the $\beta$ decay is required. The gross theory is a type of macroscopic model to describe various $\beta$ decays. It is based on sum rules of the $\beta$-decay strength function, and treats the transitions to all the final nuclear levels in a statistical manner. The gross theory has been successful in describing $\beta$ decay for the entire nuclear mass range [1-6]. The results of the gross theory provide a guide to experiments on $\beta$ decay, especially, for newly measured nuclear data of very neutron-rich nuclei, and for purely theoretical nuclear data extremely far from known nuclei. Due to its statistical treatment, the gross theory only describes macroscopic features, and the gross theory has not included a kind of of microscopic properties as spin, parity of nuclei.

In this paper, we try to introduce an effect of microscopic correction of the gross theory, and discuss

a e-mail: koura.hiroyuki@jaea.go.jp an effect on resulting half-lives. Especially, we focus on the parity-change of interchanging between groundstate neutron and proton, as a single-particle state. We estimate virtual single-particle state with a global oneparticle potential and give a suppression to the allowed transition of the $\beta$ decay. The essence of this model is on the treatment of the $\beta$-decay strength function, which corresponds to the squared matrix element of transition.

\section{Beta decay}

The decay constant of the $\beta$-decay can be divided by the types of $\beta$-decay operators, $\Omega$, and the decay constant is obtained as the sum of partial decay constants, $\lambda_{\Omega}$. If we take in to account the allowed and first forbidden transitions, the total $\beta$-decay rate is expressed as

$$
\lambda_{\beta}=\lambda_{\mathrm{F}}+\lambda_{\mathrm{G}-\mathrm{T}}+\lambda_{\text {first-forbidden }}+\cdots
$$

where the right-handed sides represents the decay rates of the Fermi transition, Gamow-Teller transition, and the first-forbidden transitions. The first two terms are the allowed transition.

Under the usual approximation, each decay rate can be written with the nuclear matrix elements, $\left|M_{\Omega}(E)\right|$, which can be calculated in the framework of the nuclear physics, and the integrated Fermi function, $f$, which represents a distortion of wave functions due to the Coulomb force. The 


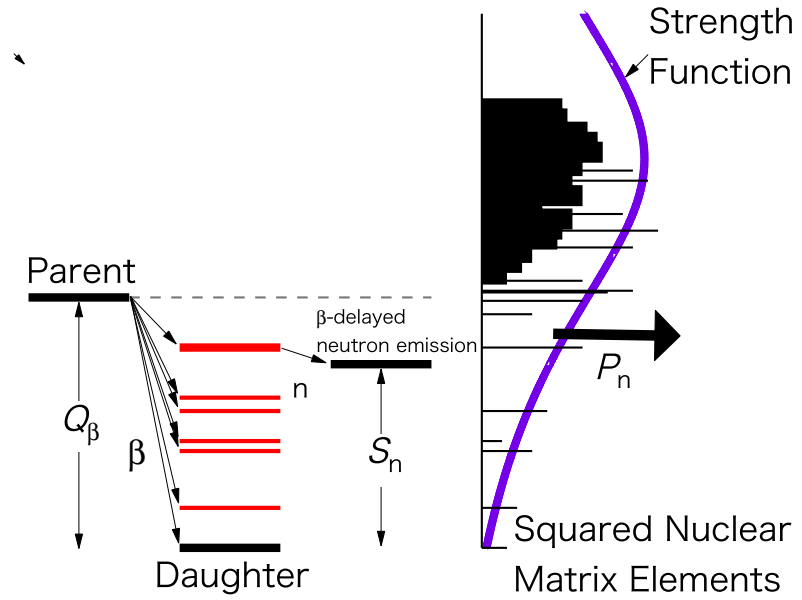

Figure 1. Schematic illustration of $\beta$ decay and delayed neutron emission.

actual expressions for allowed transition are

$$
\begin{aligned}
\lambda_{\mathrm{F}} & =\frac{m_{\mathrm{e}}^{5} c^{4}}{2 \pi^{3} \hbar^{7}}\left|g_{\mathrm{V}}\right|^{2} \int_{-Q_{\beta}}^{0}\left|M_{\mathrm{F}}(E)\right|^{2} f(-E) d E \\
\lambda_{\mathrm{GT}} & =\frac{m_{\mathrm{e}}^{5} c^{4}}{2 \pi^{3} \hbar^{7}}\left|g_{\mathrm{A}}\right|^{2} 3 \int_{-Q_{\beta}}^{0}\left|M_{\mathrm{GT}}(E)\right|^{2} f(-E) d E
\end{aligned}
$$

Here, the coefficients are composed of the coupling constant of the weak interaction and the physical constants as the mass of electron, $m_{\mathrm{e}}$, the light velocity, $c$, the Planck constant per $2 \pi, \hbar$. The coupling constant of the weak interaction has two types: the vector type as $g_{\mathrm{V}}$ and the axial vector type as $g_{\mathrm{A}}$, respectively. The integral is performed from $-Q_{\beta}$ to 0 and $Q_{\beta}$ is the total (maximum) decay energy from the ground-state of parent to daughter nuclei, or $\beta$-decay $Q$-value.

The emission of delayed neutrons is a phenomenon that accompanies $\beta$ decay. Figure 1 shows a schematic illustration of the $\beta$ decay and delayed neutron emission processes. In $\beta$ decay, a nucleus decays from the parent ground state to a particular daughter state. If the neutron separation energy for the daughter nucleus, $S_{\mathrm{n}}$, is smaller than $Q_{\beta}$, the nucleus can emit a neutron with an energy from 0 to $Q_{\beta}-S_{\mathrm{n}}$, measured from the ground state of the parent nucleus. In this case, the delayed neutron emission probability is calculated by integrating from the threshold energy to the ground-state energy of the parent nucleus:

$$
P_{\mathrm{n}}=\frac{C}{\lambda_{\beta}} \int_{-Q_{\beta}+S_{\mathrm{n}}}^{0}|M(E)|^{2} f(-E) \frac{\Gamma_{\mathrm{n}}}{\Gamma_{\mathrm{n}}+\Gamma \gamma} d E,
$$

where $C$ is a constant related to the coefficients outside the integrals in Eq. (1). The decay width $\Gamma$ is introduced to express the competition between neutron emission and gamma decay from excited states.

As shown in Eq. (1), the nuclear matrix elements and the integrated Fermi function are required for theoretical calculation of the $\beta$-decay rate and the delayed neutron emission probability. Regarding the integrated Fermi function, the numerical values can be rather easily and precisely obtained. The calculation of the nuclear matrix elements, is however, difficult because of a complexity of the nuclear many body problem with the complicated nuclear force. Historically, two type of approaches have been studied: One is the 'microscopic' approach currently known as the quasi-random phase approximation (QRPA). The other is the 'macroscopic approach' known as the gross theory (GT), discussed in the following.

\section{Gross theory}

The gross theory is constructed under a consideration on the sum of strengths of the transition from the initial state to the sum of the final states in quantum mechanics. The $\beta$ decay also obeys such a sum (and an energy-weighted sum) rule. The following is the general relation for the nuclear matrix elements of the Fermi transition as

$$
\begin{aligned}
\int_{-\infty}^{\infty}\left|M(E)_{\mathrm{F}}\right|^{2} & =1 \\
\int_{-\infty}^{\infty} E\left|M(E)_{\mathrm{F}}\right|^{2} & = \pm \Delta E_{\mathrm{C}} \\
\int_{-\infty}^{\infty} E^{2}\left|M(E)_{\mathrm{F}}\right|^{2} & ={\sigma_{\mathrm{C}}}^{2},
\end{aligned}
$$

where the $\Delta E_{\mathrm{C}}$ is the difference of the Coulomb energy between parent and daughter nuclei including the difference of masses of neutron and hydrogen, and $\sigma_{\mathrm{C}}$ is the dispersion of the nuclear matrix element contributed by the Coulomb force. For the other transitions similar relations can be obtained.

In the gross theory, a one-particle strength function, $D_{\Omega}$, is introduced, and the nuclear matrix element can be written as

$$
\left|M(E)_{\Omega}\right|^{2}=\int_{\epsilon_{\min }}^{\epsilon_{\max }} D_{\Omega}(E, \epsilon) W(E, \epsilon) \frac{d n_{1}}{d \epsilon} d \epsilon,
$$

where $\epsilon$ is the one particle energy of the decaying nucleons, and $E$ is the transition energy measured from the parent state. The function $W(E, \epsilon)$ is a weight function to take into account the Pauli exclusion principle, and $d n_{1} / d \epsilon$ the one-particle energy distribution of the decaying nucleons. The actual explicit form is given in Ref. [2]. The one-particle function, $D(E, \epsilon)$ is a smooth function and also satisfied the sum and energy-weighted sum rules as the same as for the case of $|M(E)|^{2}$. This is the essential idea of the gross theory.

\section{Improvement}

The decay constant of $\beta$ decay is expressed as Eq. (1). Actually, each transition is hindered by nuclear structure as spin and parity. In the framework of the original gross theory, there are no restrictions on the nuclear microscopic properties. All the types of transition are calculated and summed for all nuclei.

Suppose single-particle levels for neutron and proton of the ground-state at the Fermi surface. If the parities of the neutron and the proton in the ground-state is different, the allowed transition is forbidden. If the parity of surface neutron and proton are different, the allowed transition of the $\beta$ decay from ground-state to the ground-state should not occur. Figure 2 shows examples for ${ }_{49}^{131} \mathrm{In}_{82}$ and ${ }_{49}^{131} \mathrm{In}_{82}$. In the case of ${ }_{49}^{131} \mathrm{In}_{82}$, single-neutron level of the groundstate is $2 \mathrm{~d}_{3 / 2}$ with positive parity, while single-proton levels of the ground-state is $1 \mathrm{~g}_{9 / 2}$ with positive parity: 

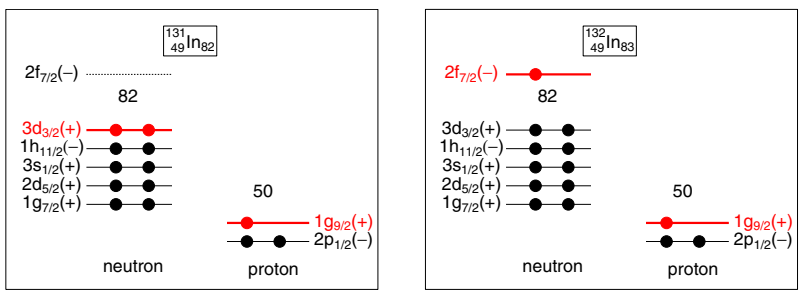

Figure 2. Schematic view of single-particle levels for ${ }_{49}^{131} \mathrm{In}_{82}$ and ${ }_{49}^{132} \mathrm{In}_{83}$. The red part represents the ground-state level.

which is the same parity. In the case of ${ }_{49}^{132} \mathrm{In}_{83}$, however, single-neutron level of the ground-state changes to $2 f_{7 / 2}$ with negative parity, and resulting the allowed transition cannot occur. In the actual $\beta$-decaying nucleus, the decay rate is the sum of the all corresponding transitions of possible levels between neutrons and protons though the ground-to-ground state is generally dominant.

We introduce such a hindrance for the allowed transition into the gross theory. We estimate the spinparity of the ground-state single-particle levels, and then treat the case of parity-mismatching between neutron and proton of the ground-state to be suppressed for the allowed transition. In the gross theory, nuclear levels are treated as the continuum with one discrete level at the Fermi surface with the Fermi gas model [2]. The nuclear matrix element is therefore composed as four parts:

$$
\begin{aligned}
\left|M_{\Omega}(E)\right|^{2} & =\left|M_{\Omega S \rightarrow 1}(E)\right|^{2}+\left|M_{\Omega S \rightarrow \mathrm{C}}(E)\right|^{2} \\
& +\left|M_{\Omega \mathrm{C} \rightarrow \mathrm{C}}(E)\right|^{2}+\left|M_{\Omega \mathrm{C} \rightarrow 1}(E)\right|^{2} .
\end{aligned}
$$

Here, ' $S$ ' represents the discrete level of neutron (the surface level), 'l' represents the discrete level of proton (the lowest level), and ' $\mathrm{C}$ ' represent the continuum of levels for the $\beta^{-}$case (see Ref. [2]). We sustain the intensity for the continuum-to-continuum component, $\left|M(E)_{\Omega \mathrm{C} \rightarrow \mathrm{C}}\right|^{2}$, and other three components are suppressed in the work. We emphasize that this treatment is applied for all nuclei: even-even, odd-A and odd-odd nuclei. Therefore considered spin and parity is different from experimental spin and parity of the ground-state for eveneven $\left(0^{+}\right.$in total), odd-odd (integer) and odd-A with large deformations.

For the estimation of spin-parity, we adopt a modified Woods-Saxon potential applied for spherical nuclei in the entire region of nuclear chart [8]. The treatment is only done for small nuclear shapes, therefore we estimate nuclear deformation from global calculation of ground-state nuclear masses. We apply the KTUY mass formula [9] for the estimation. The threshold deformation parameter is determined so as to reproduce experimental trends, and we adopt the quadruple deformation parameter $\alpha_{2}=0.05$.

Figure 3 shows the result of location of mismatching of parity in the wide nuclear mass region. The mismatching region is located along the magic number of neutrons and protons as $N \sim 82$ and $28 \leq Z \leq 60$, etc.

\section{Results}

\subsection{Half-lives}

Figure 4 shows $\beta^{-}$-decay half lives for silver to tin isotopes in the neutron-rich mass region. The region above

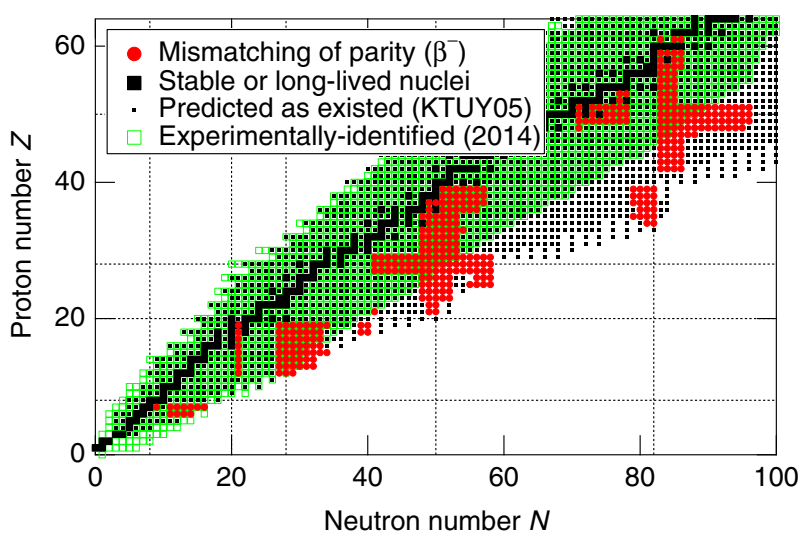

Figure 3. Mismatching of parity between ground-state neutron levels and proton levels (Red). Only $\beta^{-}$side is plotted. Filled square (Black): stable or long-lived nuclides. Open square (green): nuclides experimentally identified [7]. Dot (gray): nuclides predicted as existed by the KTUY mass model [9].

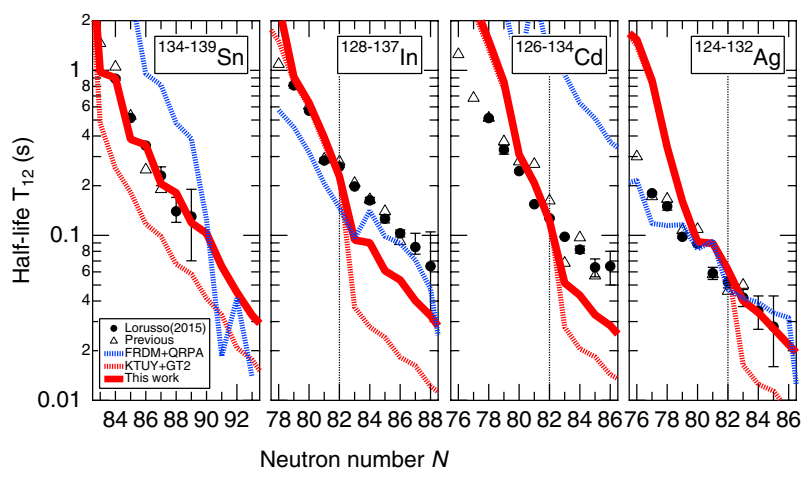

Figure 4. $\beta^{-}$-decay half lives for silver to tin isotopes in the neutron-rich mass region. Thin red line: previous gross theory [6] with the KTUY mass model [9]. Thick red line: this work. Blue dashed line: FRDM+QRPA [10]. Filled circle: experiment till 2015. Open triangle: experiment in 2015 [11].

$N=82$, half-lives from the previous gross theory [6] with the KTUY mass model [9] (thin line) systematically slopes down and underestimates. These isotopes are located in the mismatching region as shown in Fig. 3. The improved gross theory (thick lines) enhances half-lives in this region and consequently gives gradual slopes and follows experimental trend. The result of FRDM + QRPA calculation [10] is also plotted, and this results rather diverge from experimental trend and sometimes gives a staggering line.

\subsection{Delayed neutron probabilities}

Delayed neutron probabilities can be calculated with Eq. (1). Figure 5 shows delayed neutron probabilities, $P_{\mathrm{n}}$, for selected nuclei which are related to the paritymismatching. The gross theory generally gives smooth trends along isotopes. The improved ones give rather lower values than original gross theory. The improvement in this work does not significantly change delayed neutron probabilities as compared with half-lives. Experiments generally give gradual increases. The result of FRDM + QRPA calculation [10] sometimes gives different trends to the gross theory as shown in the figure. 

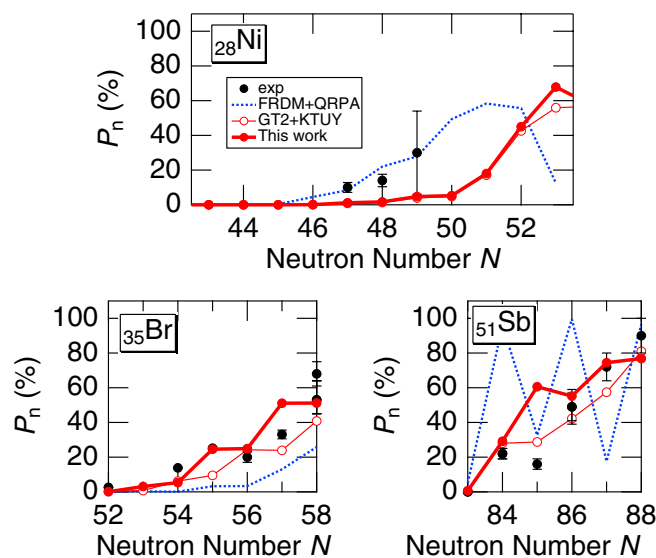

Figure 5. Delayed neutron probabilities for nickel, antimony, and bromine isotopes. Thin red line: previous gross theory [6] with the KTUY mass model [9]. Thick red line: this work. Blue dashed line: FRDM+QRPA [10]. Filled black circle with error bar: experiment.

\subsection{Programming code}

With this work, we have described the development of a programming code of the gross theory including the improved parts. Using this code, $\beta$-decay halflives, delayed-neutron probabilities, averaged beta and gamma ray energies, neutrino spectrums can be calculated. Moreover, various versions of the gross theory as Refs. [1-6] can be selected, and also the functional form of one-particle strength function can be chosen. After preparation finished, this code can be released for the nuclear data community.

\section{Summary}

In conclusion, the gross theory of $\beta$-decay is improved on the parity-changes in the allowed transitions. In this treatment, the nuclear matrix elements are suppressed when the parity between single neutron and proton is different for the allowed transition. The assignment of parity is done by using the Woods-Saxon type singleparticle potential and the KTUY nuclear mass model.
The discrepancies from experimental half-lives, which appeared in the vicinity with the magic number of neutrons and protons, are improved. Despite the improvement, delayed neutron probabilities change slightly to the lower.

In the treatment of this work, the sum rule of $\beta$-decay squared matrix element is not considered, and therefore the sum of intensities over the entire energy range may lower than one expected for the corresponding nuclei. This point will deal with in the next work.

Present study is the result of "Comprehensive study of delayedneutron yields for accurate evaluation of kinetics of high-burn up reactors" entrusted to Tokyo Institute of Technology by the Ministry of Education, Culture, Sports, Science and Technology of Japan (MEXT).

\section{References}

[1] K. Takahashi, M. Yamada, Prog. Theor. Phys. 41, 1470 (1969)

[2] S. Koyama, K. Takahashi, M. Yamada, Prog. Theor. Phys. 44, 663 (1970)

[3] K. Takahashi, M. Yamada, T. Kondoh, Atom. Dat. Nucl. Dat. Tab. 12, 101 (1973)

[4] T. Kondoh, T. Tachibana, M. Yamada., Prog. Theor. Phys. 74, 708 (1985)

[5] T. Tachibana, M. Yamada, Y. Yoshida, Prog. Theor. Phys. 84, 641 (1990)

[6] T. Tachibana, M. Yamada, Proc. Inc. Conf. on Exotic Nuclei and Atomic Masses, ENAM95, (Editions Frontueres, Gif-sur-Yvette, 2015), p. 763, and references therein

[7] H. Koura, J. Katakura, T. Tachibana, F. Minato, Chart of the Nuclides 2014, Japanese Nuclear Data Committee and Nuclear Data Center, Japan Atomic Energy Agency (2015)

[8] H. Koura, M. Yamada, Nucl. Phys. A 671, 96 (2000)

[9] H. Koura, T. Tachibana, M. Uno, M. Yamada, Prog. Theor. Phys. 113, 305 (2004)

[10] P. Möller, J.R. Nix, K.-L. Kratz, Atom. Dat. Nucl. Dat. Tab. 66, 131 (1997)

[11] G. Lurusso et al., Phys. Rev. Lett. 114, 192501 (2015) 